\section{Therapeutic Clowning in Pediatric Practice: A Novel Concept to Think About in India}

Therapeutic or medical clowning is a new concept across various healthcare settings around the world [1]. It is a paramedical practice in which clowns are associated with healthcare system to mitigate anxiety, stress, fear and sadness in admitted patients, thereby augmenting the healing process [2]. They create a more positive and constructive hospital environment and trust between patients and medical teams. Research has concluded that medical clowns have a significantly positive effect in adults [3]. A consistent observation has been seen that clowns are always appreciated by pediatric patients [4].

Idea of medical clowning was conceptualized by Michael Christensen in 1986, in the United States. At a physiological level, laughing stimulates release of endorphins modulating immune system. Laughing also replaces negative feeling with positive ones at and emotional level. Clowning distracts the child from the current situation at the cognitive level. Socially, laughing stimulates better interaction between children and health care personnel $[4,5]$.

This practice is still nascent at present in India. Sir JJ Hospital Mumbai has begun with medical clowning in pediatric wards recently. As a pediatrician, our primary responsibility is better health and quality of life of our pediatric patients, and hence, this novel idea of therapeutic clowning is worth trying, especially to begin with vaccination sessions. Further research is warranted to replicate its results in the Indian settings.

Published Online: September 05, 2020: PII: S097475591600239.

Shahid AKHTAR Siddiqui* and MUKESH Vir Singh Department of Pediatrics, SN Children Hospital, MLN Medical College, Allahabad, Uttar Pradesh, India.

*sha.akht@yahoo.com

\section{REFERENCES}

1. Finlay F, Baverstock A, Lenton S. Therapeutic clowning in paediatric practice. Clin Child Psychol Psychiatry. 2014; 19:596-605.

2. Nuttman-Shwartz O, Scheyer R, Tzioni H. Medical clowning: even adults deserve a dream. Soc Work Health Care. 2010;49:581-98.

3. van Venrooij LT, Barnhoorn PC. Hospital clowning: A paediatrician's view. Eur J Pediatr. 2017;176:19197.

4. Barkmann C, Siem A-K, Wessolowski N, SchulteMarkwort M. Clowning as a supportive measure in paediatrics - A survey of clowns, parents and nursing staff. BMC Pediatr. 2013;13:166.

5. Bennet MP, Lengacher C. Humor and laughter may influence health: III. Laughter and health outcomes. Evid Based Complement Alternat Med 2007; 5:37-40.

\section{Multisystem Inflammatory Syndrome in Children (MIS-C) - Recent Updates}

We read the very timely article by Bhat, et al. [1] providing valuable insights into clinical epidemiology of multisystem inflammatory syndrome in children (MIS-C). We comment on the recent evidence to complement the information provided.

Recent clinical guidelines by American College of Rheumatology (ACR) elaborate on the most appropriate diagnostic and therapeutic steps for MIS-C at the present time, advising inflammatory markers and cytokine panel testing [2]. There is noteworthy discordance in interleukin levels of IL-1, IL-6 and IL-10 among patients with Kawasaki disease (KD) $v s$ MIS-C [3]. While IL-1 is the main mediator of coronary artery inflammation in $\mathrm{KD}$, inflammatory process in MIS-C is predominantly driven by IL- 6 and IL-10, which may play a role in the myocardial dysfunction and higher severity of the 2019$\mathrm{nCoV}$ infection [4].
We concur with the authors that the role for specific cytokine blockade including use of biologics in MIS-C is still lacking. The ACR guidelines advice immunomodulatory therapy for all severe/critical MIS-C patients with shock, significant respiratory distress, neurologic changes, dehydration, or features of KD. IVIG and glucocorticoid remain first line agents either alone or in combination. Anakinra is safe in severe infections among children with hyper-inflammatory syndromes. Although tocilizumab is effective in reducing mortality and ICU admission in patients with severe COVID19 pneumonia [2], the clinical evidence is insufficient regarding its efficacy and safety for COVID-19 because of concerns regarding risk of secondary bacterial and fungal infections [5]. Aspirin (3-5 mg/kg/day) should be used in patients with MIS-C and KD-like features and/or thrombocytosis and continued until normali-zation of platelet count and confirmed normal coronary arteries at $\geq 4$ weeks after diagnosis. Anticoagulation with enoxaparin should be added in patients with coronary artery aneurysm and $Z$ score $\geq 10.0$ or an ejection fraction (EF) $<35 \%$ [2], but despite benefits, strategy based evidence is required due to high risk of hemorrhagic events or complications.

With the availability of these guidelines a standardized treatment plan for MIS-C involving multidisciplinary care 